\title{
Comparison of in vitro methods for the production of Paenibacillus larvae endospores
}

\author{
Israel Alvarado ${ }^{\mathrm{a}}$, Michelle M. Elekonich ${ }^{\mathrm{a} \wedge}$, Ernesto Abel-Santos ${ }^{\mathrm{b}}$, Helen J. Wing ${ }^{\text {a }}$ \\ ${ }^{a}$ School of Life Sciences, 4505 South Maryland Parkway, Bldg WHI, M/S 4004, \\ University of Nevada, Las Vegas, Las Vegas, NV 89154-4004 \\ ${ }^{b}$ Department of Chemistry, 4505 South Maryland Parkway, Bldg CHE, M/S 4003, \\ University of Nevada, Las Vegas, Las Vegas, NV 89154-4003 \\ ^Permanent Address: National Science Foundation, 4201 Wilson Blvd. Arlington, VA 22230 \\ ${ }^{\S}$ Corresponding author
}

Email addresses:

IA: alvara95@unlv.nevada.edu
ME: melekoni@nsf.gov

EAS: ernesto.abelsantos@unlv.edu

§HJW: helen.wing@unlv.edu Tel: 7028955382 


\section{Abstract}

Paenibacillus larvae endospores are the infectious particles of the honey bee brood disease, American Foulbrood. We demonstrate that our previously published protocol (Alvarado et al., 2013) consistently yields higher numbers and purer preparations of $P$. larvae endospores, than previously described protocols, regardless of the strain tested (B-3650, B-3554 or B-3685).

Keywords: Paenibacillus larvae, American Foulbrood disease, spore production, honey bee 
The Gram-positive, spore-forming bacterium Paenibacillus larvae is the causal agent of American Foulbrood disease (White, 1907). Honey bee larvae become infected when contaminated brood food containing infectious spores is fed to larvae by the "nurse" bees, which, like all adult honey bees, are resistant to AFB (Wilson, 1971). The spores of $P$. larvae are highly resilient and can remain viable for at least 35 years (Haseman, 1961). Eradication of $P$. larvae spores from infected hives is commonly achieved by burning the entire hive and all associated equipment (Calderone, 2001; Matheson, 1993), but this highly destructive solution increases the economic losses associated with this disease. Targeted biological approaches that eradicate spores from equipment have significant potential, but the development of such strategies requires large scale and pure preparations of $P$. larvae endospores.

While three procedures for in vitro sporulation of $P$. larvae have been commonly used ((Dingman, 1983; Dingman and Stahly, 1983; Genersch et al., 2005) and recently described in (de Graaf et al., 2013)), these methods do not yield large enough numbers of spores of sufficient purity for studies that seek to identify chemical inhibitors and/or germinants of $P$. larvae spores (Akoachere et al., 2007; Alvarez et al., 2010; Howerton et al., 2011; Powell, 1950; Ramirez and Abel-Santos, 2010). Consequently, a new method was developed ["UNLV”; (Alvarado et al., 2013)]. Here, we present a comparison of the UNLV in vitro sporulation protocol (Alvarado et al., 2013) with a protocol (described in de Graaf et al., 2013) that has traditionally yielded the highest number of spores (Dingman, The Connecticut Agricultural Experiment Station; personal communication). Notably, in both protocols spores are prepared from solid growth medium.

To account for strain differences, three different $P$. larvae strains were used in this comparative study (Table 1). In the first protocol (de Graaf et al., 2013), spores were harvested from MYPGP agar inoculated with $P$. larvae. Since "sporulation efficiency can decline when high numbers of colonies are present on a plate" (de Graaf et al., 2013), cultures were serially diluted using MYPGP broth to obtain both low (50-200) and high (1,000-5,000) CFU counts per 
MYPGP plate prior to spore preparation. This allowed a thorough comparison of this protocol (de Graaf et al., 2013) with the other (Alvarado et al., 2013). After 7 days of incubation, spores were removed from MYPGP plates by gently scraping and washing the agar surface with sterile water three times. The spore suspension was concentrated via centrifugation at 12000 $\mathrm{rpm} / 19800 \mathrm{~g}$ at $4{ }^{\circ} \mathrm{C}$ for 15 minutes, the supernatant was discarded and the remaining pellet was resuspended in sterile ice-cold water. Additional centrifugation and pellet resuspension steps were repeated four times to clean spores. Spore stocks produced in this manner were stored in sterile distilled water at $4^{\circ} \mathrm{C}$.

TABLE 1: Strains of $P$. larvae used.

\begin{tabular}{cccc} 
Species & $\begin{array}{c}\text { ERIC } \\
\text { classification }^{\text {a }}\end{array}$ & Strain number $^{\mathbf{b}}$ & Isolation \\
\hline P. larvae & $I$ & NRRL B-3650 & $\begin{array}{c}\text { Isolated from diseased honey bee, RE } \\
\text { Gordon }\end{array}$ \\
P. larvae & $I$ & NRRL B-3554 & $\begin{array}{c}\text { Isolated from diseased honey bee larvae, } \\
\text { NRRL }\end{array}$ \\
P. larvae & IV & NRRL B-3685 & Powdery scale of honey bee larvae, RE \\
& /ATCC-49843 & Gordon
\end{tabular}

${ }^{a}$ Evaluated at UNLV by Enterobacterial repetitive intergenic consensus sequence-based PCR (Genersch et al., 2006). ' Strain numbers sourced from the American Tissue Culture Collection (www.atcc.org) are prefixed with ATCC, those from the Agriculture Research Services (ARS) Culture Collection Database Server (nrrl.ncaur.usda.gov; formerly known as the Northern Regional Research Laboratories, NRRL) are prefixed with NRRL.

In contrast, the UNLV method utilized Tryptic Soy Agar (TSA) plates (supplied by Becton, Dickinson and Company) inoculated with $200 \mu$ of an exponentially growing culture (about $1.2 \mathrm{x}$ $10^{8}$ colony forming units, CFUs) that were incubated for 7 days in a $5 \% \mathrm{CO}_{2}$ incubator at $37^{\circ} \mathrm{C}$ to produce lawns of $P$. larvae (Alvarado et al., 2013). The resulting spore-containing bacterial lawns were collected by flooding plates with ice-cold deionized water. Spores were pelleted by centrifugation at $8000 \mathrm{rpm} / 8820 \mathrm{~g}$ at $4{ }^{\circ} \mathrm{C}$ for 5 minutes and resuspended in fresh deionized water. After three washing steps, the spores were separated from their vegetative and partially sporulated forms by centrifugation at $11500 \mathrm{rpm} / 18200 \mathrm{~g}$ at $4{ }^{\circ} \mathrm{C}$ for 35 minutes through a 20 - 
$50 \%$ HistoDenz $^{\mathrm{TM}}$ (Sigma Aldrich) gradient. Spore pellets were washed five times with sterile distilled water and stored at $4^{\circ} \mathrm{C}$.

To measure the concentration of viable spores in each spore stock, counts of heat resistant colony forming units were calculated from MYPGP agar plates (Dingman, 1983). Briefly, spore stocks were normalized to an $\mathrm{OD}_{580}$ of 0.2 , heated at $68^{\circ} \mathrm{C}$ for 15 minutes, diluted in water and plated onto MYPGP agar. Plates bearing about 30-300 colonies per plate were used to calculate the number of heat-resistant spores in each stock (Dingman, 1983). Assays were performed in triplicate and average CFUs, standard deviations and statistically significant differences $(p<0.05)$ for all pairs of mean heat resistant spore counts per stock were determined using a Student's one-tailed $t$ test assuming equal variances.

As expected, spore yields varied from strain to strain regardless of the protocol was used. Consistent with previous findings, yields from B-3554 and B-3650 were higher when spores were harvested from plates with lower CFU density than higher; a trend not seen for B-3685. Importantly, regardless of the strain tested or whether the spores were isolated from either low or high density plates, the UNLV protocol (Alvarado et al., 2013) yielded significantly higher numbers of spores than the established MYPGP plate method (de Graaf et al., 2013) (more than 385-fold higher for strain B-3554, 2.6-fold higher for strain B-3650 and 142-fold higher for strain B-3685; see Figure 1A), allowing us to conclude that the UNLV protocol routinely yields higher numbers of viable spores than the de Graaf protocol, regardless of the $P$. larvae strain used.

The purity of spore stocks was next determined by microscopic analysis. Briefly, $10 \mu$ of each spore stock was smeared onto a glass slide, air dried, and observed after Schaeffer-Fulton staining (Schaeffer and Fulton, 1933). At least 10 images of each spore stock were randomly acquired and three random images were analyzed using image processing software (ImageJ, $\mathrm{NIH}$ ). The purity of spore preparations viewed in these images was expressed as: (number of 
spores/number of spores and vegetative cells) $X 100$. Statistically significant differences $(p<$ 0.05 ) for all pairs of mean spore preparations or percent purity were determined using a Student's one-tailed $t$ test assuming equal variances.

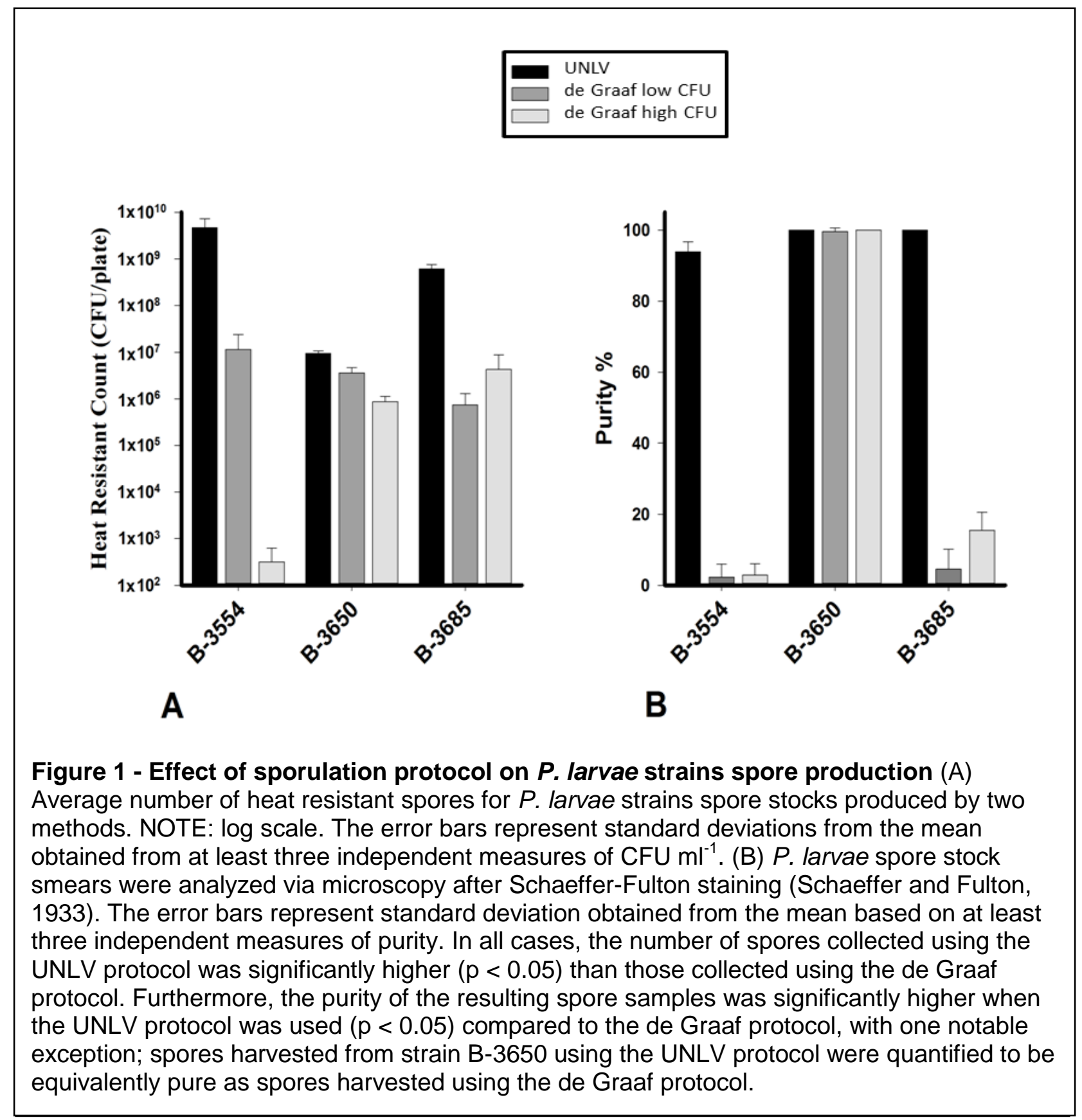

This analysis revealed that spore stocks prepared from strains B-3554 and B-3685 using the de Graaf protocols consisted of less than $20 \%$ spores (Figure 1B). Furthermore, spores 
prepared using this protocol, were frequently contaminated with cell debris, although the amount was difficult to quantify by microscopy. For example, even though spore stocks generated from strain B-3650 were found to be $100 \%$ pure (did not contain any vegetative cells) cell debris could still be observed by microscopy (Figure 2A). These results indicate that four washes used in the previously reported protocols (de Graaf et al., 2013; Dingman and Stahly, 1983) were not sufficient to remove cell debris from the spore stocks. While it is possible to remove vegetative cells from spore stocks by heating, this approach is not recommended because muropeptides released from vegetative cells have been shown to trigger spore germination (Shah et al., 2008). In the UNLV protocol, this problem was solved by using a HistoDenz ${ }^{\mathrm{TM}}$ density gradient.

This approach separated the spores from vegetative cells and cell debris and yielded virtually

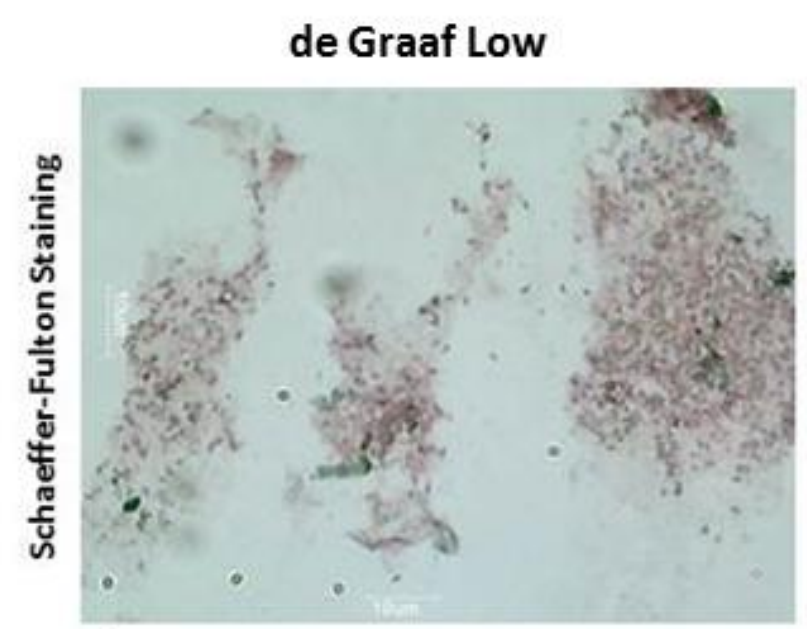

A

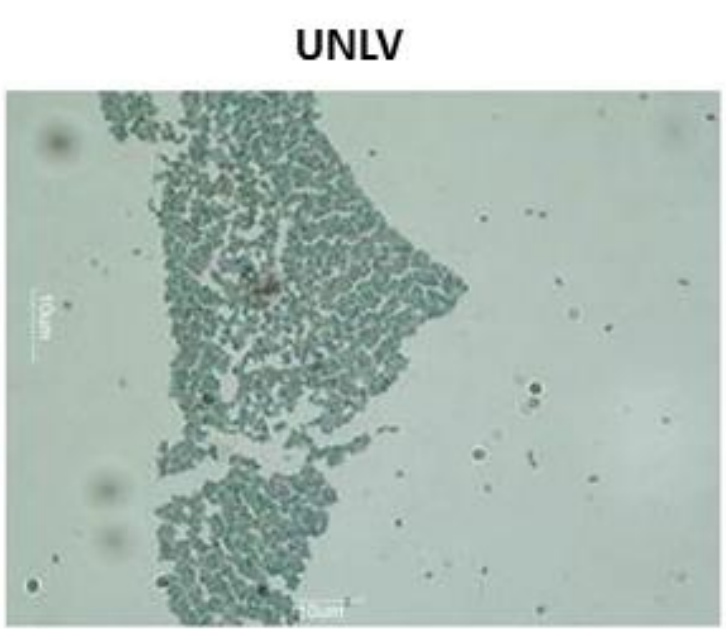

B

Figure 2 - Purity of spore stocks determined by microscopic analysis (A) Representative image of spore stocks stained using the Schaeffer-Fulton method (Schaeffer and Fulton, 1933) prepared for strain B-3650 (A) using the de Graaf protocol or (B) the UNLV protocol. Cell debris retained safranin-O dye while spores retained malachite green dye. The scale bars indicates $10 \mu \mathrm{m}$.

pure preparations of spores, regardless of the strain tested (Figure 2B).

In sum, this comparative analysis demonstrates that the UNLV in vitro sporulation method is more effective at generating higher numbers of spores with greater purity than the established in vitro sporulation protocol (de Graaf et al., 2013; Dingman and Stahly, 1983). The use of a 
commercially available rich medium, TSA, facilitated spore production and streamlined media production compared to other protocols (de Graaf et al., 2013; Dingman, 1983; Dingman and Stahly, 1983; Genersch et al., 2005) and the use of a centrifugation density gradient ensured that a pure stock was obtained. Although, we acknowledge that similar results might be obtained if the numbers of plates and tubes were increased while following older protocols (Dingman, 1983; Dingman and Stahly, 1983; Genersch et al., 2005), a differential centrifugation step would still be required to increase the purity of the spores generated. This comparative analysis highlights the strengths and weaknesses of the currently published protocols for the in vitro preparation of $P$. larvae spores from solid medium (Alvarado et al., 2013; de Graaf et al., 2013), and as such will help those requiring large scale and pure preparations of $P$. larvae endospores and possibly other bacterial endospores.

\section{Acknowledgements}

Permission to submit this work was granted by Diane Yost who had copyrighted an earlier draft of this manuscript as a chapter in her Master's thesis. We thank M. Picker and Dr. N. Griffin for critical reading of the manuscript and editorial comments. IA was supported by a UNLV Hermsen Fellowship, and UNLV Strategic Plan Graduate Research Assistantship (SPGRA) awarded to EAS and MME. This work was supported by USDA Grant NEVR-2010-03755. MME was also supported by the NSF Independent Research and Development program. The views expressed in this publication belong to the authors and do not necessarily reflect the views of the National Science Foundation. 


\section{References}

Akoachere, M., Squires, R.C., Nour, A.M., Angelov, L., Brojatsch, J., and Abel-Santos, E. (2007). Identification of an in vivo inhibitor of Bacillus anthracis spore germination. J Biol Chem 282, $12112-12118$.

Alvarado, I., Phui, A., Elekonich, M.M., and Abel-Santos, E. (2013). Requirements for in vitro germination of Paenibacillus larvae spores. J Bacteriol 195, 1005-1011.

Alvarez, Z., Lee, K., and Abel-Santos, E. (2010). Testing nucleoside analogues as inhibitors of Bacillus anthracis spore germination in vitro and in macrophage cell culture. Antimicrob Agents Chemother 54, 5329-5336.

Calderone, N. (2001). Management of Honey Bee Brood Diseases. Part 1: Identification \& Treatment. American Foulbrood, 2001. In The Bee Files

de Graaf, D.C., Alippi, A.M., Antunez, K., Aronstein, K.A., Budge, G., De Koker, D., De Smet, L., Dingman, D.W., Evans, J.D., Foster, L.J., et al. (2013). Standard methods for American foulbrood research. J Apicult Res 52.

Dingman, D.W. (1983). Bacillus larvae: Parameters Involved with Sporulation and Characteristics of Two Bacteriophages. In Biological Sciences (Ann Arbor: The University of lowa), pp. 281.

Dingman, D.W., and Stahly, D.P. (1983). Medium Promoting Sporulation of Bacillus larvae and Metabolism of Medium Components. Appl Environ Microbiol 46, 860-869.

Genersch, E., Ashiralieva, A., and Fries, I. (2005). Strain- and genotype-specific differences in virulence of Paenibacillus larvae subsp. larvae, a bacterial pathogen causing American foulbrood disease in honeybees. Appl Environ Microbiol 71, 7551-7555.

Genersch, E., Forsgren, E., Pentikainen, J., Ashiralieva, A., Rauch, S., Kilwinski, J., and Fries, I. (2006). Reclassification of Paenibacillus larvae subsp. pulvifaciens and Paenibacillus larvae subsp. larvae as Paenibacillus larvae without subspecies differentiation. Int J Syst Evol Microbiol 56, 501-511.

Haseman, L. (1961). How long can spores of American foulbrood live? American Bee Journal 101, 298-299.

Howerton, A., Ramirez, N., and Abel-Santos, E. (2011). Mapping interactions between germinants and Clostridium difficile spores. J Bacteriol 193, 274-282.

Matheson, A. (1993). World Bee Health Report. Bee World 74, 176-212.

Powell, J.F. (1950). Factors affecting the germination of thick suspensions of Bacillus subtilis spores in L-alanine solution. Journal of general microbiology 4, 330-338.

Ramirez, N., and Abel-Santos, E. (2010). Requirements for germination of Clostridium sordellii spores in vitro. J Bacteriol 192, 418-425.

Schaeffer, A.B., and Fulton, M.D. (1933). A Simplified Method of Staining Endospores. Science 77, 194.

Shah, I.M., Laaberki, M.H., Popham, D.L., and Dworkin, J. (2008). A eukaryotic-like Ser/Thr kinase signals bacteria to exit dormancy in response to peptidoglycan fragments. Cell $135,486-$ 496.

White, G.F. (1907). The Cause of American Foulbrood. 1907., B.o.E. United States Department of Agriculture, ed. (Washington D.C.: Washington D.C., Government Printing Office). Wilson, W.T. (1971). Resistance to American foulbrood in honey bees. XI: Fate of Bacillus larvae spores ingested by adults. J Invertebr Pathol 17, 247-255. 